\title{
New era of treatment with biologics in rheumatology - is it time to shift paradigms in treatment with biologics?
}

\author{
Anna Felis-Giemza \\ Department of Systemic Connective Tissue Diseases, Biological Therapy Center, National Institute of Geriatrics, \\ Rheumatology and Rehabilitation, Warsaw, Poland
}

In recent years due to a continuous progress of genetic engineering resulting in production of monoclonal antibodies (mAb) new class of drugs was introduced to revolutionize treatment of many diseases, especially in autoimmunology and oncology. Initially, when treatment with mAbs was an absolute novelty, it provoked uncertainty during the recruitment for the treatment and its subsequent monitoring. Currently, when we know much more about their effects, we can monitor the therapy more efficiently. Indications for the use of biologics in rheumatic diseases have also widened. We currently use biologic therapy for patients not only with rheumatoid arthritis (RA), spondyloarthropathies but also with vasculitis and connective tissue diseases such as systemic lupus erythematosus, poli/dermatomyositis and Sjögren's syndrome.

Thus, biologic drugs have become a common and often necessary therapeutic option in rheumatology. Suitable procedures have been developed to screen patients for latent and potentially serious infections, e.g. tuberculosis (tbc) and there are chemoprophylaxis schemes. Some vaccinations are recommended before the initiation of biologic treatment. Hepatitis $C$ infection has ceased to be a challenge for rheumatologists when effective antiviral drugs were introduced [1].

Observational studies and case reports of women exposed to tumor necrosis factor (TNF) inhibitors during pregnancy suggest that their pregnancy outcomes are similar to those in women with RA not treated with biologics, including rates of preterm birth, spontaneous miscarriage, and congenital anomalies [2]. It has also been shown that the treatment during pregnancy with certain biologic drugs do not carry a significant risk of congenital disorders. Additionally, the inhibition of an active inflammatory process can be beneficial for both a mother and a child as well [2-4].
The approach to the perioperative management in patients treated with biologics has also recently been changed. It has been agreed for most drugs that there is no need for a break prior to surgery for multiple (3-5) half-lives of the drug. In recent American College of Rheumatology/American Association of Hip and Knee Surgeons (ACR/AAHKS) recommendations elective total hip or total knee arthroplasty is suggested to be performed at the end of dosing cycle (plus 1 week) for each specific biologic medication [5]. Restart of the biologic treatment is recommended at minimum 14 days after surgery in the absence of wound healing issues, surgical site or systemic infections [5].

It is still true, that a general risk of infections during biologic therapy is increased. Serious infections, sepsis, tuberculosis and cases of opportunistic infections, including fatalities, have been reported with the use of TNF blocking agents. Other serious infections seen in clinical trials and in the clinical practice - including pneumonia, pyelonephritis, septic arthritis and septicemia - can also lead to fatal outcomes. On the other hand it is still underestimated, that there is even greater risk of infection in case of the corticosteroid use. Thus, close monitoring should be performed in the RA patients treated with biologics and glucocorticosteroids, mainly in elderly patients and those with a low total lymphocyte count at the beginning of biologic treatment [6].

It is well known, that the combined therapy of biologics with methotrexate is recommended because it increases the potential of biologic drugs and inhibits their loss of efficacy due to the formation of anti-drug antibodies. There are also observations about beneficial combinations of novel agents. Recently, articles have been published, based on the research of small cohorts of patients, in which a combination therapy with tofacitinib was introduced in the event of the lack or loss 
of efficacy of treatment with biologics, TNF inhibitors in particular. In those studies deaths or serious adverse effects were not reported.[7, 8].

The risk of cancer caused by anti-TNF use has been extensively studied over time and the results are showing no evidence of overall increased risk. It is highly possible that anti-TNF is associated with incidence of non-melanoma skin cancers, but it is probably comparable to that created by the use of to classic disease-modifying antirheumatic drugs. Even for patients with recent malignancies recommendations point to rituximab and reassure, that this treatment is safe and should be preferred $[1,9]$.

To conclude, in current management of inflammatory rheumatic diseases, biological drugs have a well established position. One can undoubtedly expect further development of this type of therapies. Through the introduction of biosimilars biologics are also becoming more widely available. They pose less uncertainties as experience in their use is constantly growing. It altogether shifts our paradigm on many of the earlier restrictions, such as pregnancy, infections and surgery.

The author declares no conflict of interest.

\section{References}

1. Singh JA, Furst DE, Bharat A, et al. 2012 update of the 2008 American College of Rheumatology recommendations for the use of disease-modifying antirheumatic drugs and biologic agents in the treatment of rheumatoid arthritis. Arthritis Care Res (Hoboken) 2012; 64: 625-639.
2. Marchioni RM, Lichtenstein GR. Tumor necrosis factor-alpha inhibitor therapy and fetal risk: A systematic literature review. World J Gastroenterol 2013; 19: 2591-2602.

3. Flint J, Panchal S, Hurrell A, et al. BSR and BHPR guideline on prescribing drugs in pregnancy and breastfeeding - Part I: standard and biologic disease-modifying anti-rheumatic drugs and corticosteroids. Rheumatology (Oxford) 2016; 55: 1693-1697.

4. Skorpen CG, Hoeltzenbein M, Tincani A, et al. The EULAR points to consider for use of antirheumatic drugs before pregnancy, and during pregnancy and lactation. Ann Rheum Dis 2016; 75: 795-810.

5. Goodman SM, Springer B, Guyatt G, et al. ACR/AAHKS Guidelines for Perioperative Management Special Article. 2017 American College of Rheumatology/American Association of Hip and Knee Surgeons Guideline for the Perioperative Management of Antirheumatic Medication in Patients With Rheumatic Diseases Undergoing Elective Total Hip or Total Knee Arthroplasty. Arthritis Rheumatol 2017; 69: 1538-1551.

6. Leon L, Peñuelas M, Candel FJ, et al. Indicator opportunistic infections after biological treatment in rheumatoid arthritis, 10 years follow-up in a real-world setting. Ther Adv Musculoskelet Dis 2019; 11: 1759720X19878004.

7. Singh JA, Hossain A, Tanjong Ghogomu E, et al. Biologics or tofacitinib for people with rheumatoid arthritis naive to methotrexate: a systematic review and network meta-analysis. Cochrane Database Syst Rev 2016; 5: CD012183.

8. Barroso N, Furst DE. A Case Series on Patients on Tofacitinib in Combination with a Biologic [abstract]. Arthritis Rheumatol 2016; 68 (Suppl 10). https://acrabstracts.org/abstract/a-caseseries-on-patients-on-tofacitinib-in-combination-with-a-biologic/ (accessed 27.10.2019).

9. Seror R, Mariette X. Malignancy and the Risks of Biologic Therapies Current Status. Rheum Dis Clin North Am 2017; 43: 43-64. 